\title{
Cosmic ray effect on the X-ray Trigger Telescope of UFFO/Lomonosov using YSO scintillation crystal array in space
}

M. B. Kim ${ }^{1 *}$, S. Jeong ${ }^{1,2}$, H. M. Jeong ${ }^{1}$, V. Leonov', J. Lee ${ }^{1}$, I.H. Park ${ }^{1 \S}$, A.M.

Amelushkin $^{3}$, V.O. Barinova ${ }^{3}$, A.V. Bogomolov ${ }^{3}$, V.V. Bogomolov ${ }^{3}$, S. Brandt ${ }^{4}$, C. Budtz-Jørgensen ${ }^{4}$, A.J. Castro-Tirado ${ }^{2,5}$, P. Chen ${ }^{6,7}$, P. Connell, , G. Garipov ${ }^{3}$, E.S. ${\text { Gorbovskoy }{ }^{3} \text {, N.L. Dzhioeva }{ }^{3} \text {, C. Eyles }}^{8}$, M.-H. A. Huang ${ }^{9}$, A.F. lyudin ${ }^{3}$, V.V. $K_{\text {Kalegaev }}^{3}$, P.S. Kasarjan ${ }^{3}$, J.E. Kim ${ }^{1}$, V.G. Kornilov ${ }^{3}$, E.A. Kuznetsova ${ }^{3}$, H. Lim ${ }^{1}$, V.M. Lipunov', T.-C. Liu', I.N. Myagkova ${ }^{3}$, J.W. Nam ${ }^{6,7}$, M.I. Panasyuk ${ }^{3}$, M.I. Panchenko $^{3}$, V.L. Petrov ${ }^{3}$, A.V. Prokhorov ${ }^{3}$, V. Reglero ${ }^{8}$, J. Ripa ${ }^{7}$, J. M. Rodrigo ${ }^{8}$, A.N. Shustova ${ }^{3}$, S.I. Svertilov ${ }^{3}$, N.V Tyurina ${ }^{3}$, I.V. Yashin ${ }^{3}$

\footnotetext{
${ }^{1}$ Department of Physics, Sungkyunkwan University, 2066, Seobu-ro, Jangan-gu, Suwon, Gyeonggi-do, 16419, Korea

${ }^{2}$ Instituto de Astrofisica de Andalucia ZAA-CSIC,P. O. Box 03004, E-18080 Granada, Spain

${ }^{3}$ Skobeltsyn Institute of Nuclear Physics of Lomonosov, Moscow State University, Leninskie Gory, Moscow, 119234, Russia

${ }^{4}$ National Space Institute, Technical University of Denmark, 2800 Kgs. Lyngby, Denmark

${ }^{5}$ Departamento de Ingeniería de Sistemas y Automática, Universidad de Málaga, Spain

${ }^{6}$ Leung Center for Cosmology and Particle Astrophysics, National Taiwan University, 1 Roosevelt Road, Taipei, 10617, Taiwan

${ }^{7}$ Department of Physics, National Taiwan University, 1 Roosevelt Road, Taipei, 10617, Taiwan

${ }^{8}$ GACE, Edif. de Centros de Investigacion, Universidad de Valencia, Burjassot, E-46100 Valencia, Spain

${ }^{9}$ Department of Energy Engineering, National United University, 1, Lienda,36003 Miaoli, Taiwan
}

\section{E-mail: ilpark@skku.edu}

UFFO Burst Alert and Trigger telescope (UBAT) is the X-ray trigger telescope of $\mathrm{UFFO/Lomonosov} \mathrm{to} \mathrm{localize} \mathrm{X-ray} \mathrm{source} \mathrm{with} \mathrm{coded} \mathrm{mask} \mathrm{method} \mathrm{and} \mathrm{X-ray} \mathrm{detector.} \mathrm{Its} \mathrm{X-}$ ray detector is made up of $368 \times 8$ pixels Yttrium OxyorthoSilicate (Y2SiO5:Ce, YSO) scintillation crystal arrays and 36 64-channel Multi-Anode PhotoMultiplier Tubes (MAPMTs) for space mission. Its effective detection area is $161 \mathrm{~cm}^{2}$ and energy range is several $\mathrm{keV}$ to $150 \mathrm{keV}$. It was successfully launched in April 28, 2016. In several calibration run, we got several X-ray background data. We already knew X-ray background flux is 2-3 counts $/ \mathrm{cm}^{2} / \mathrm{sec}$ in space. However our X-ray background data shows approximately 7-8 times higher than what we know. There are many candidates to explain high X-ray background count in space. One of candidates

*Speaker

${ }^{\S}$ Corresponding author 
is cosmic ray. We will report cosmic ray effect on the X-ray detector using YSO scintillation crystal arrays in space.

35th International Cosmic Ray Conference - ICRC2017

10-20 July, 2017

Bexco, Busan, Korea 


\section{Introduction}

We developed Ultra-Fast Flash Observatory (UFFO) - pathfinder to observe Gamma-Ray Bursts (GRBs)[1]. It is made up of data acquisition system (UDAQ) and 2 telescopes.

One is Slewing Mirror Telescope (SMT) which consisits of slewing mirror systsem, $100 \mathrm{~mm}$ aperture Ritchey-Chrétien telescope, Intensified CCD (ICCD) and readout electronics. Its purpose is GRB targeting and tracking within a few seeconds with slewing mirror system and measuring UV/optical signal from GRBs[2,3].

The other is UFFO Burst Alert and Trigger telescope (UBAT) which localizes GRB with coded mask method and gives GRB location to SMT [4]. It consists of external structure, socalled hopper, X-ray detector and electronics. X-ray detector is made up of $368 \times 8$ pixels Yttrium OxyorthoSilicate $\left(\mathrm{Y}_{2} \mathrm{SiO}_{5}\right.$ :Ce, YSO) scintillation crystal arrays and 36 64-channel Multi-Anode PhotoMultiplier Tubes (MAPMTs). YSO scintillation crystal array has many advantages. First it dosen't have intrincsic background. It means we can detect low energy X-ray withount internal noise. And it has high light yield and high spatial resolution by adopting pixelized crystals. An effective detection area of UBAT flight model is $161 \mathrm{~cm}^{2}$ and energy range is several $\mathrm{keV}$ to 150 $\mathrm{keV}$.

UFFO-pathfinder/Lmonosov was successfully launched on April 28, 2016 and we are calibrating our instrument in space.
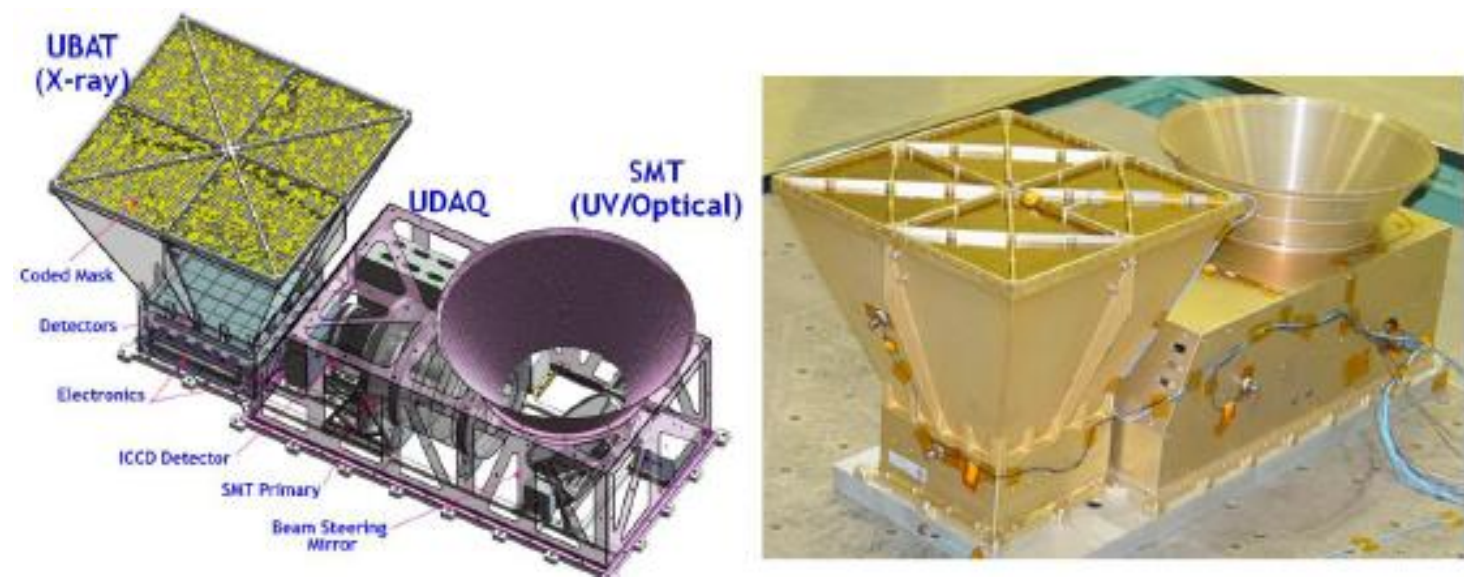

Figure 1: A composition of UFFO-pathfinder (left) and assembled flight model (right)[1]

\section{Performance of UBAT in space}

After launch, we started telescope calibration in space. During that time, the X-ray trigger telescope (UBAT) took several data.

We checked detector performance through diffused X-ray data analysis. UBAT takes 700 frames data and it is divided 5 sessions. We are able to adjust exposure time of each session by commend. Table 1 shows default value of number of frame and exposure time in each session. We set the default value of exposure time and took a data on July 5, 2016. Figure 2 shows its results. 
It is 1 of 700 frames X-ray data. A white color means X-ray was detected and a black color means no X-ray on detector. (a) and (b) were set $100 \mathrm{~ms}$ exposure time and (c) and (d) were set $500 \mathrm{~ms}$ exposure time. (c) and (d) have many X-ray hits in comparison with (a) and (b). It means our session setting is working well.

Table 1: Default value of number of frame and exposure time

\begin{tabular}{ccc}
\hline $\begin{array}{c}\text { Session } \\
\text { Number }\end{array}$ & $\begin{array}{c}\text { Number of } \\
\text { frame }\end{array}$ & $\begin{array}{c}\text { Exposure time per } \\
\text { 1 frame (sec) }\end{array}$ \\
\hline 1 & 200 & 0.1 \\
\hline 2 & 300 & 0.1 \\
\hline 3 & 100 & 0.5 \\
\hline 4 & 50 & 0.5 \\
\hline Total & 700 & 0.5 \\
\hline
\end{tabular}

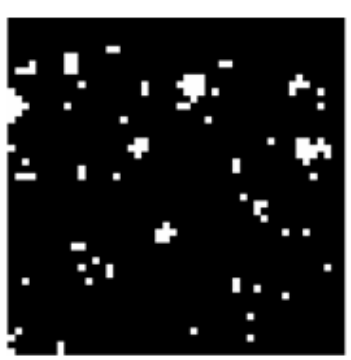

(a)

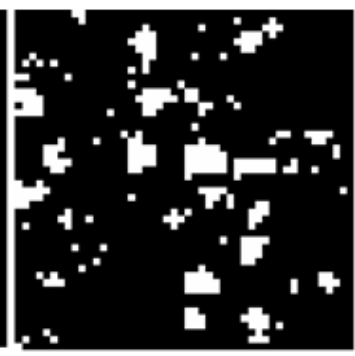

(b)

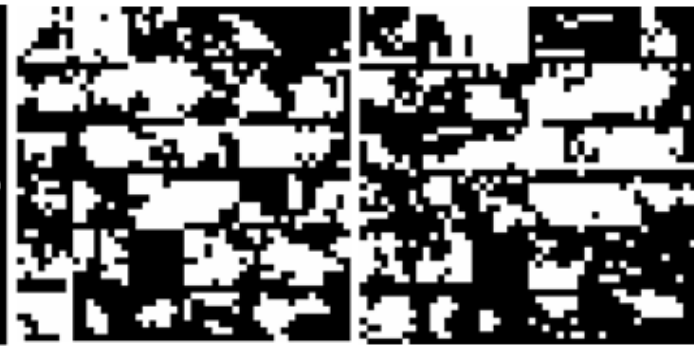

(c) (d)

Figure 2: X-ray telescope(UBAT) detector data in space, 48channel $\times 48$ channel. (a) and (b) are $100 \mathrm{mse}$ c exposure time and (c) and (d)are 500msec exposure time.

In Figure 3, red points were divided 2 groups because of exposure time setting. We set 100msec exposure time from data taking start to 50 seconds and $500 \mathrm{msec}$ exposure time 51 seconds to 150 seconds. Also, we calculated count rate using this data. It is approximately 21.3 $\mathrm{cnts} / \mathrm{cm}^{2} / \mathrm{sec}$. We already known counts of diffused X-ray background is $2 \sim 3 \mathrm{cnts} / \mathrm{cm}^{2} / \mathrm{sec}$ up to $15 \mathrm{keV}$. This value is from swift which is NASA mission to observe GRB [5]. Our value is about 7 times higher than well-known value. We suggest 3 candidates of reason which are crosstalk effect, low energy sensitivity and cosmic ray effect. In section 3 , we discuss cosmic ray effect on the UBAT using beam test results. 


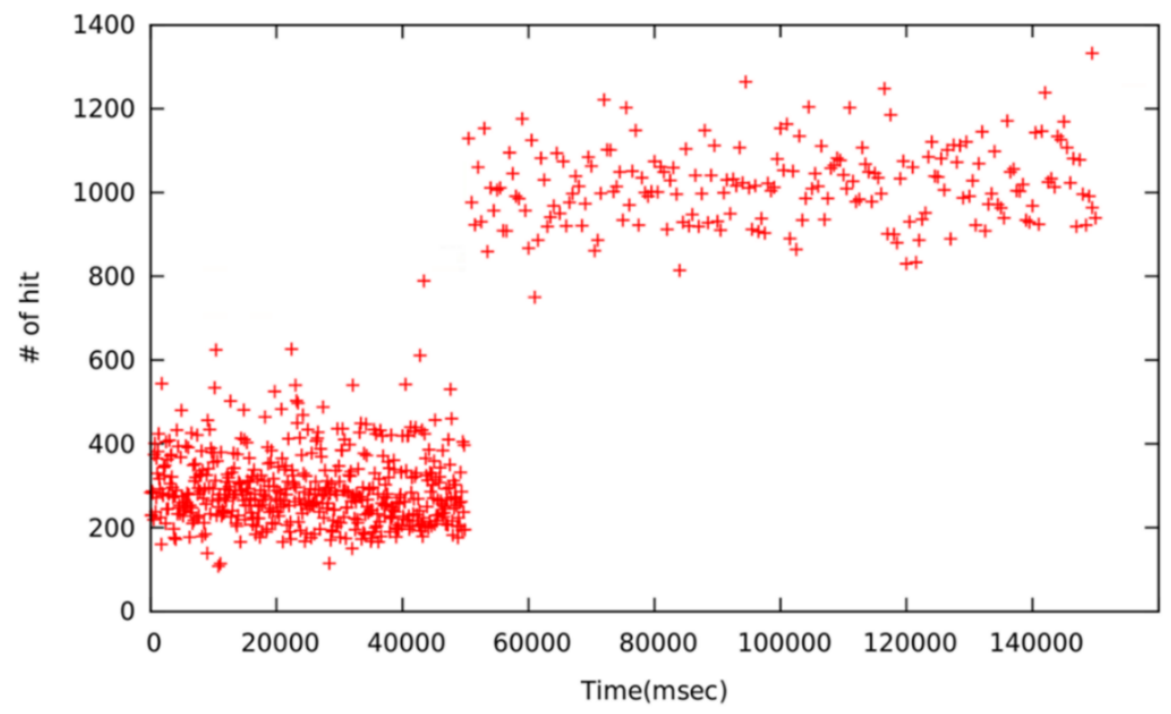

Figure 3: Number of hit of each frame on July 5,2016 data

\section{Cosmic ray effect}

\subsection{CERN beam test with mini UBAT detector}

We tested our X-ray detector with heavy incident heavy ion beam at European Organization for Nuclear Research (CERN) to confirm X-ray detector of UBAT resopose when comsic ray pass through UBAT. We prepared a mini X-ray detector of UBAT for ground test. It consists of only one analog electronics and 2 YSO scintillation crystals and MAPMTs set (see Figure 4).

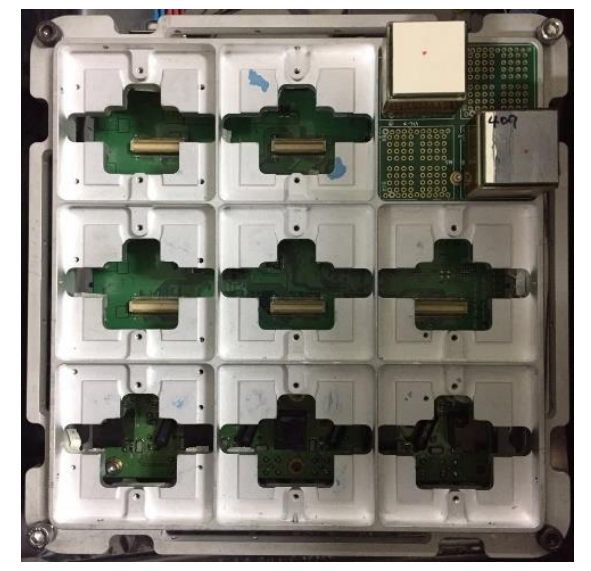

Figure 4: The mini X-ray detector of UBAT for ground test. There is an analog electronics, 2 YSO scintillation crystals and MAPMTs set on right-upper side of the UBAT detector structure.

We used $\mathrm{H} 4$ beam line at CERN. Heavy ions have a momentum of $60 \mathrm{GeV} / \mathrm{c}$ per nucleon and $\mathrm{A} / \mathrm{Z}=2.0$. We put $20 \mathrm{~cm}$ thickness of lead between beam line and our detector to produce fragmented particles like cosmic ray. And we made dark box to protect X-ray detector from light and put X-ray detector in the dark box. It was placed on the table in front of beam line. Test facility and set-up shown in Figure 5. 


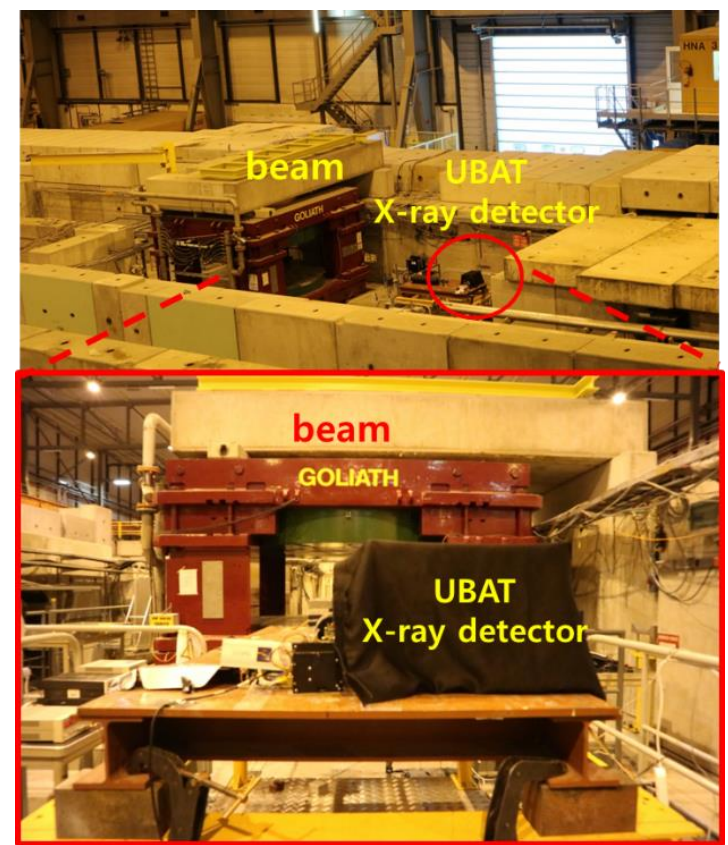

Figure 5: Test set up at $\mathrm{H} 4$ beam line of CERN. The dark box which includes X-ray detector is placed in front of beam line.

UBAT has $6 \times 6$ MAPMT and YSO scintillation crystal sets. One square is one set and mini UBAT has only 2 sets, $(1,5)$ and $(2,6)$ location. We set exposure time is $6 \mathrm{msec}$ for all frames of data, total duration time of a data is 4.2 seconds. When we took a data without any beam in the ground, we didn't see big clusters in each frame. However, we can see big clusters with heavy ion beam in Figure 6 and almost frames have big clusters. It means any charged particles make cluster on our detector and cosmic rays can make clusters.
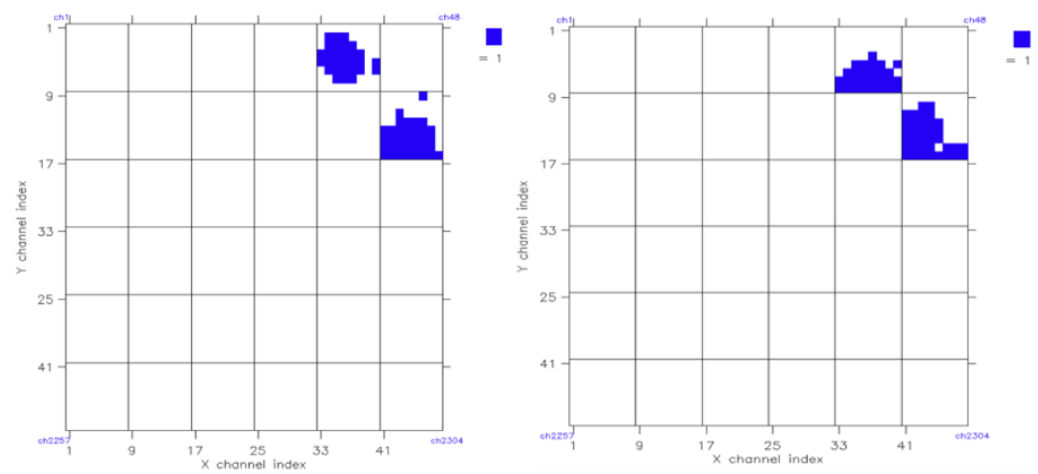

Figure 6: Each is one of frames response from beam test. It takes $6 \mathrm{msec}$ to take a frame data. UBAT has $6 \times 6$ MAPMTs originally but mini UBAT has only 2 MAPMTs. Its locations are $(1,5)$ and $(2,6)$. Blue color is response for any particles and white color is no response. 


\subsection{Cosmic ray effect on UBAT in space}

UFFO-pathfinder took several diffused X-ray background data during calibration in space. Each data has different exposure time setting. Therefore, we checked $1 \mathrm{msec}$ per a frame data of all data because if exposure time is long, some small clusters gather and make big cluster and our minimum settable exposure time is $1 \mathrm{msec}$ for $\mathrm{X}$-ray detection. Also, when a cluster includes more than $30 \mathrm{X}$-ray hitting pixels, we defined it is made by cosmic ray. Figure 7 shows diffused X-ray background data from space. In diffused X-ray background data by our detector, approximately $5 \%$ of total frames have big cluster like Figure 7 . As we ignore frames which include big cluster and calculate count rate again, we are able to get about $12.3 \mathrm{cnts} / \mathrm{cm}^{2} / \mathrm{sec}$. This value is 1.7 times less than previous value.
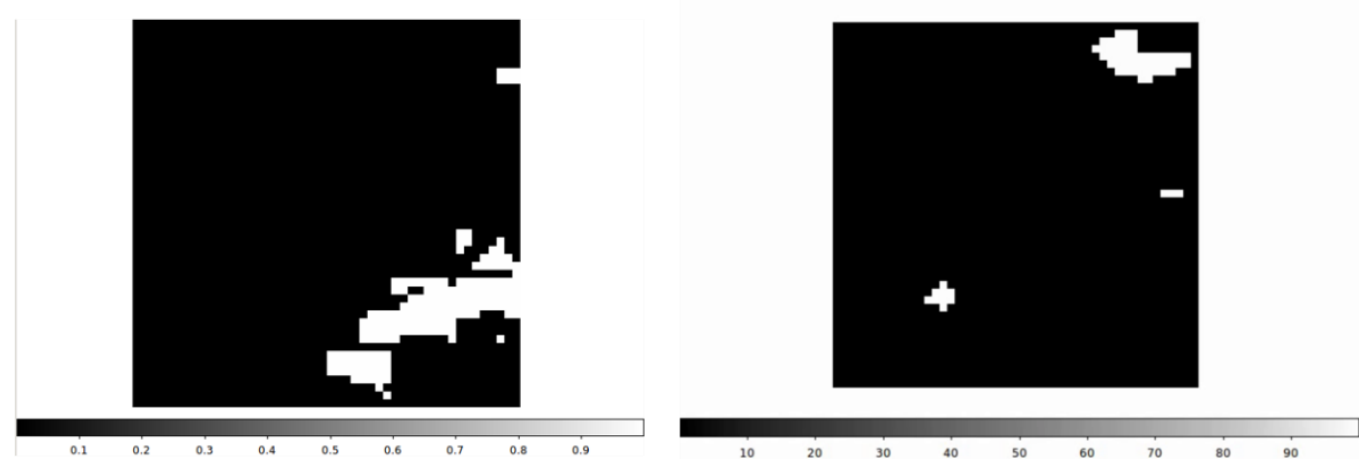

Figure 7. $48 \times 48$ channels X-ray hitmap in space. A white color is X-ray hitting channel and black is no Xray channel. Left: One of 700 frames data on Sep.29,2016. Exposure time is 1msec. Right: One of 700 frames data Nov.24, 2016. Exposure time is $1 \mathrm{msec}$.

\section{Conclusions}

UFFO Burst Alert \& Trigger telescope (UBAT) is X-ray telescope of UFFO/Lomonosov to detect X-ray and localize Gamma-Ray Bursts (GRBs). Its detector was made up of YSO scintillation crystal arrays and MAPMTs. They are the first materials as X-ray detector in space, we confirmed that they are suitable for space mission.

After launch, UBAT got several diffused X-ray background data in space during calibration. $\mathrm{X}$-ray count rate is 7 times higher than as we expected from swift results. We assumed several candidates to find reason of high count rate. One of candidates is cosmic ray effect on UBAT because X-ray hitmap of several data frames has big clusters it cannot be from X-ray. We tested our X-ray detector at CERN to confirm cosmic ray effect on ours. We set proper beam and test condition to make charged particles. We confirm that charged particles made big clusters on our detector.

UBAT doesn't have any cosmic ray counter to distinguish it. So, as hitmap of a frame has big clusters which are connected more than 30 pixels, we defined it is from cosmic ray. We calculated how many big clusters are detected. Approximately $5 \%$ of all frames of $1 \mathrm{msec}$ exposure time have it. As we remove big cluster, X-ray count rate is $\sim 12.3 \mathrm{cnts} / \mathrm{cm}^{2} / \mathrm{sec}$. Cosmic ray effect reduce about $40 \%$ of X-ray count. In our following paper, we will report for low energy sensitivity and crosstalk effect on UBAT. 


\section{Acknowledgements}

This work was supported by NRF (National Research Foundation of Korea) Grant funded by the Korean Government (NRF-2015-Fostering Core Leaders of the Future Basic Science Program/Global Ph.D. Fellowship Program)

\section{References}

[1] I.H. Park et al., Ultra-Fast Flash Observatory for the observation of early photons from gamma-ray bursts, New Journal of Physics 15.2 (2013): 023031

[2] S. Jeong et al., Slewing mirror telescope optics for the early observation of UV/optical photons from gamma-ray bursts, Optical Express, 21(2):2263-2278 (2013).

[3] J. E. Kim et al., Readout of the UFFO slewing mirror telescope to detect uv/optical photons from gamma-ray bursts. JINST (2013)

[4] J. E. Kim et al., Design and implementation of the UFFO burst alert and trigger telescope, SPIE Astronomical Telescopes Instrumentation. International Society for Optics and Photonics (2012)

[5] M. Ajello et al., Cosmic X-ray Background and Earth Albedo Spectra with SwiftBAT, The American Astronomical Society (2008) 